\title{
Modern View on Demodecosis Diagnostic
}

\section{Kubanov Alexey, Ph.D ${ }^{1}$., Gallyamova Yulia, Ph.D²., Kravchenko Anzhela, Ph.D*}

${ }^{1}$ Head of department of dermatovenereology and cosmetology of Russian medical academy of continuous professional education of the Ministry of Healthcare of the Russian Federation, Russia

${ }^{2}$ Professor of department of dermatovenereology and cosmetology of Russian medical academy of continuous professional education of the Ministry of Healthcare of the Russian Federation, Russia

${ }^{3}$ student of department of dermatovenereology and cosmetology of Russian medical academy of continuous professional education of the Ministry of Healthcare of the Russian Federation, Russia. angkravchenko@gmail.com

*Corresponding Author: Kravchenko Anzhela, Ph.D., student of department of dermatovenereology and cosmetology of Russian medical academy of continuous professional education of the Ministry of Healthcare of the Russian Federation, Russia.

\section{Abstract}

Demodex mites are one of the most frequently identified microorganisms in patients with acne and rosacea. There are a lot of methods for detecting of Demodex mites exist. It is always relevance to find and introduce new diagnostic methods with modern characteristics. This article considers different methods of Demodex diagnostic. The literature review represents all advantages and disadvantages of all methods.

Keywords: confocal laser scanning in vivo microscopy; Demodex mites; skin scrapers microscopy; standardized surface skin biopsy; histology; dermatoscopy.

\section{INTRODUCTION}

Nowadays it is known that Demodex mites play a role in the development of facial dermatoses, accompanied by the appearance of papulopustular elements (acne, rosacea) [1-4]. Moreover, the presence of mites on the skin of a person is not always accompanied by the development of a clinical picture of the disease. It can be argued that Demodex mites are conditionally pathogenic representatives of the microflora of human skin [5].

The diagnosis of "Demodecosis" is possible only after laboratory diagnosis, during which Demodex mites will be found. Accordingly, clinically diagnose "Demodecosis" is not possible. The procedure for diagnosing demodecosis in a laboratory is simple and informative, although, at the present time, it is far from ideal and requires further development.

\section{LIGHT MICROSCOPY OF SKIN SCRAPERS}

Laboratory diagnostics is based on the compilation of an acarogram by counting larvae, nymphs, eggs and adults and is an objective diagnostic criterion. To evaluate the ongoing therapy, repeated acarograms are performed to count the number and determine the activity of mites. The criterion for mites`activity is the number of more than 5 adults, larvae or eggs per $\mathrm{cm}^{2}$. If only products of vital activity and empty egg shells are found in the scrapings, a second study is carried out. During the treatment, the movement of Demodex mites to areas untreated with acaricidal agents was noted. In such cases, most often, mites are localized at the edge of the scalp. Since, often, mites are localized in the depth of the sebaceous-hair follicles; they can not always be detected. In this regard, light microscopy of scrapings is not a highly informative method and does not prove the absence of mites [6].

Mites can be found on the damaged area of the skin when extracting the contents of the follicle or removing eyelashes/eyebrows without damaging the hair follicles. The test material is placed on a slide with $10 \%$ alkaline solution $(\mathrm{KOH}, \mathrm{NaOH})$, covered with a slide and viewed under a small magnification of the microscope. In addition to alkali as an immersion medium, it is possible to use $50 \%$ glycerol, immersion oil. The advantage of the technique is the ability to 
analyze the presence of mites not only on the surface of the skin but also directly in the sebaceous glands. Here there is another problem - it is not always possible to get to the mites in the depths of the sebaceous glands. The disadvantage is also the traumatization of the epithelium, sometimes with the appearance of bleeding, examination of small lesions, the relative morbidity of the procedure, the discomfort of patients after epilation and the time consuming [7].

\section{STANDARDIZED SURFACE SKIN BIOPSY}

A standardized surface skin biopsy (SSSB) is available $[6,8,9]$. A drop of cyanocrylate glue (BF-6, sulfacrylate) is applied to the non-fatty coverslip, and then glued to the affected surface for 1 minute. On a drop of cyanoacrylate, a $1 \mathrm{~cm}^{2}$ area is designated for further counting the tick-load quantitative load. After removal, an alkali solution is applied, covered with a cover glass and viewed under a microscope at a small magnification [7]. Disadvantages of the diagnosis can be that the mites do not always adhere to the surface of the drop of glue, and sometimes they can not be detected with microscopy due to the fact that between the mites and the glue there can be cornified epithelial cells or sebum. Therefore, before the study, the skin should be degreased with ether [7].

\section{Histology}

Another method for diagnosing demodecosis is to perform a skin biopsy followed by histology of the preparations obtained. For this purpose, puncture (punch) or excision (scalpel) method takes a small area of skin; fix it during the day with $10 \%$ neutral formalin solution, seal with paraffin and stain with hematoxylin-eosin. Histological examination gives a lot of advantages. In particular, you can completely see the sebaceous gland and the surrounding areas. The main disadvantage of the method is the traumatization of the skin with the formation of the scar, as well as the inability to examine the large surface of the skin [10].

\section{DERMATOSCOPY}

As a diagnostic tool for detecting mites of the genus Demodex Segal R. et al. (2010) suggested using a dermatoscope. When using dermatoscopy as a method of research in demodecosis, the author describes three pathognomonic characteristic features: "demodex tails" - in the form of whitish threads 1-3 mm long with localization at the mouth of the hair follicles or excretory ducts of the sebaceous glands; dilated follicles with the presence of gray or brown plugs inside, as well as horizontally arranged dilated blood vessels [11].

\section{Confocal LASER ScanNing in Vivo MicRoscope}

Marvin Minsky in 1957 patented a "scanning microscope with a two-stage focusing" (the term "confocal" - based on the conjugation of foci). If a mercury or xenon lamp is used as a fluorescent light source in conventional fluorescence microscopes, then in modern confocal microscopes it is a laser. For the first time, a laser in confocal microscopy was used by Davidovich P. in 1969. A diode laser was used as a source of monochromatic and coherent light in modern confocal microscopes in order to more accurately operate the optical system of the microscope, reduce the number of glare in the images, and improve the focusing of the light beam. A focused laser beam illuminates a specific point of the skin [12]. Due to a certain microscope device, the back focus of the condenser, where the "confocal" diaphragm of the photodetector is installed, coincides with the front focus of the lens; images are obtained from a very thin layer of the object - "optical sections". The work of the confocal laser in vivo scanning microscope is based primarily on the ability of various skin structures to refract laser radiation, thus obtaining images of epidermal and dermal layers [13] and assessing the condition of the skin vessels and the dermis fibres [14]. Confocal laser scanning in vivo microscopy is a new method for studying the structure of the skin in the form of pictures of white-grey-black shades. Images of structures are achieved by refracting light from the focal region and the deeper the area, the higher laser power is required [15]. Melanocytes and keratinocytes in the pictures look bright white, air, serous fluid - black [16]. Confocal laser scanning in vivo microscopy allows you to determine the thickness and visualize different layers of the skin. Thus, the method provides additional information on the composition and structure of the skin [17]. In ophthalmology, it is possible to visualize changes in meibomian glands in the form of enlargement or obstruction, the presence of inflammatory infiltrates, and also to detect mites of the genus Demodex [18]. The method of confocal laser scanning in vivo microscopy can be equated to a histological examination of the skin with the advantage that the study is performed non-invasively [13]. According to different data, depending on the 
nosology, the sensitivity of the method is $83-91 \%$, the specificity is $95-99 \%[19,20,21]$.

The use of confocal laser scanning in vivo microscopy in dermatology is today considered to be one of the most promising methods, despite the fact that it has a number of disadvantages (obtaining relatively surface images up to $200 \mu \mathrm{m}$, which limits the possibility of studying deeper layers of the skin, images, the high cost of equipment and its operation and, as a consequence, inaccessibility for a larger number of dermatologists) [14].

In comparison with conventional light microscopy, the advantages of the method are high-contrast images with high resolution, their three-dimensional reconstruction, digital processing of the data obtained, as well as the possibility of conducting research in dynamics and, directly, at the patient's bed due to the portability of the device $[14,15]$. One of the advantages of the method is the ability to detect and quantify Demodex folliculorum on the face skin of patients with rosacea, count the number of mites and follicles per unit area [22]. Sattler E.S. et al. (2012), examining the skin of patients with rosacea, described the presence of Demodex in the form of rounded or long conical structures [22]. Kojima T. et al., (2011) demonstrated the use of confocal laser scanning in vivo microscopy for the diagnosis of eye demodecosis [23]. The authors managed to find ticks in the terminal of the bulb of the eyelashes, inflammatory infiltrates around the meibomian glands and conjunctiva.

Thus, according to the scientific literature, confocal laser scanning in vivo microscopy is a non-invasive and rapid method for detecting Demodex mites, exceeding the standard methods of investigation [24].

Despite the sufficient amount of scientific material on the study of dermatoses accompanied by papulopustular eruptions, it is important to search for non-traumatic and reliable methods of assessing skin changes. One of the modern methods is the use of a confocal laser scanning in vivo microscope. Confocal laser scanning in vivo microscopy is an innovative method whose advantages are non-invasiveness and high informativeness. This method for the study of papulopustular dermatoses and detection of mites was not previously used, although the use of non-invasive techniques is always considered to be a priority in medicine.
The available methods for diagnosing demodecosis do not meet the requirements of modern medicine, they do not guarantee the absolute reliability of the test results, and they are often traumatic and not objective. In this case, an alternative direction in modern instrumental diagnostics can be confocal laser scanning in vivo microscopy, which will identify mites of the genus Demodex non-invasively with a high percentage of reliability.

\section{REFERENCES}

[1] Holmes AD. Potential role of microorganisms in the pathogenesis of rosacea. J Amer Acad Dermatol. 2013; 69(6):1025-1032

[2] Jarmuda S, O'Reilly N, Zaba R, Jakubowicz O, Szkaradkiewicz A, Kavanagh K. Potential role of Demodex mites and bacteria in the induction of rosacea. J Med Microbiol. 2012; 61(Pt 11):15041510

[3] O’Reilly N, Menezes N, Kavanagh K. Positive correlation between serum immunoreactivity to Demodex-associated Bacillus proteins and erythematotelangiectatic rosacea. Brit J Derm. 2012; 167(5):1032-1036

[4] Zhao YE, Guo N, Xun M, Xu JR, Wang M, Wang DL. Sociodemographic characteristics and risk factor analysis of Demodex infestation (Acari: Demodicidae). J Zhejiang Univ Sci B. 2011; 12(12):998-1007

[5] Baima B, Sticherling M. Demodicidosis revisited. Acta Derm Venereol. 2002; 82:3-6

[6] Crawford GH, Pelle MT, James WD. Rosacea: Etiology, pathogenesis, and subtype classification. J Am Acad Dermatol. 2004; 51:327-44

[7] Forton F, Song M. Limitations of standardized skin surface biopsy in measurement of the density of Demodex folliculorum. A case report. Br J Dermatol. 1998; 139:697-700

[8] Bonnar E, Eustace P, Powell FC. The Demodex mite population in rosacea. J Am Acad Dermatol. 1993; 28:443-8

[9] Forton F, Seys B. Density of Demodex folliculorum in rosacea: a case-control study using standardized skin-surface biopsy. $\mathrm{Br} \mathrm{J}$ Dermatol. 1993; 128(6):650-659 
Modern View on Demodecosis Diagnostic

[10] Morrison WB, DeNicola DB. Advantages and disadvantages of cytology and histopathology for the diagnosis of cancer. Semin Vet Med Surg (Small Anim). 1993; 8(4):222-7

[11] Segal R, Mimouni D, Feuerman H, Pagovitz O, David M. Dermoscopy as a diagnostic tool in demodicidosis. Int J Dermatol. 2010; 49(9):1018-23

[12] Psaty EL, Halpern AC. Current and emerging technologies in melanoma diagnosis: the state of the art. Clin Dermatol. 2009; 27(1):35-45

[13] Rajadhyaksha M. Confocal Reflectance Microscopy: Diagnosis of Skin Cancer Without Biopsy? Frontiersof Engineering. 1998:24-33

[14] Nwaneshiudu A, Kuschal C, Sakamoto FH, Anderson RR, Schwarzenberger K, Young RC. Introduction to Confocal Microscopy. J of Invest Dermatol. 2012; 132(12):e3

[15] Hofmann-Wellenhof R, Pellacani G, Malvehy J, Soyer HP. Reflectance confocal microscopy for skin diseases. Springler. Berlin. 2012

[16] Kubanova AA, Chikin VV, Shtirshneider YY, Katunina OR. Confocal laser scanning microscopy in vivo for diagnosing melanocytic skin neoplasms. Vest dermatol venerol. 2014; (3):85-94

[17] Neerken S, Gerald W, Lucassen GW, Lenderink E, Nuijs TAM. In vivo imaging of human skin: a comparison of optical coherence tomography and confocal laser scanning microscopy. Biomedicine VII. 2003; 299

[18] Messmer EM, Torres SE, Mackert MI, Zapp DM, Kampik A. In vivo confocal microscopy in blepharitis. Klin Monbl Augenheilkd. 2005; 222 (11):894-900

[19] Nori S, Rius-Diaz F, Cuevas J, Goldgeier M, Jaen P, Torres A, González S. Sensitivity and specificity of reflectance-mode confocal microscopy for in vivo diagnosis of basal cell carcinoma: a multicenter study. J Am Acad Dermatol. 2004; 51(6): 923-930

[20] Gerger A, Koller S, Kern T, Massone C, Steigner K, Richtig E, Kerl H, Smolle J. Diagnostic applicability of in vivo confocal laser scanning microscopy in melanocytic skin tumors. J Invest Dermatol. 2005; 124(3): 493-498

[21] Gerger A, Koller S, Weger W, Richtig E, Kerl H, et al. Sensitivity and specificity of confocal laserscanning microscopy for in vivo diagnosis of malignant skin tumors. Cancer. 2006; 107(1): 193-200

[22] Sattler EC, Maier T, Hoffmann VS, Hegyi J, Ruzicka T, Berking C. Noninvasive in vivo detection and quantification of Demodex mites by confocal laser scanning microscopy. Br J Dermatol. 2012; 167(5):1042-7

[23] Kojima T, Ishida R, Sato EA, Kawakita T, Ibrahim OM, Matsumoto Y, Kaido M, Dogru M, Tsubota K. In vivo evaluation of ocular demodicosis using laser scanning confocal microscopy. Invest Ophthalmol Vis Sci. 2011; 52(1):565-9

[24] Turgut Erdemir A, Gurel MS, Koku-Aksu AE, Bilgin KF, Incel P, Kutlu-Haytoglu NS, Falay T. Reflectance confocal microscopy vs standardized skin surface biopsy for measuring the density of Demodex mites. Skin Res Technol. 2014; 20(4):435-9

Citation: Kubanov Alexey, Gallyamova Yulia, Kravchenko Anzhela. Modern View on Demodecosis Diagnostic. Archives of Dermatology and Skin Care. 2018; 1(1): 14-17.

Copyright: (C) 2018 Kubanov Alexey, Gallyamova Yulia, Kravchenko Anzhela. This is an open access article distributed under the Creative Commons Attribution License, which permits unrestricted use, distribution, and reproduction in any medium, provided the original work is properly cited. 